\title{
Validation of a PTB force-balanced piston gauge primary pressure standard
}

\author{
Ahmed S. Hashad ${ }^{1}$, Wladimir Sabuga ${ }^{2}$, Sven Ehlers², Thomas Bock ${ }^{3}$ \\ ${ }^{1}$ National Institute for Standards (NIS), Giza, Egypt (Guest researcher at PTB Germany) \\ 2 Physikalisch-Technische Bundesanstalt (PTB), Braunschweig, Germany \\ 3 Physikalisch-Technische Bundesanstalt (PTB), Berlin, Germany
}

\begin{abstract}
Experimental methods using different pressure standards were applied to verify theoretical results obtained for the effective area of a piston-cylinder assembly (PCA) and for pressures measured with a force-balanced piston gauge (FPG). The theoretical effective area was based on the PCA's dimensional properties defined by the diameter, straightness and roundness measurements of the piston and cylinder, derived from gas-flow modelling, using principles of rarefied gas dynamics and presented as two values, one for absolute and the other for gauge-pressure operation mode. Both values have a relative standard uncertainty of $5 \cdot 10^{-6}$. The experimental methods chosen were designed to cover the entire operating pressure range of the FPG from $3 \mathrm{~Pa}$ to $15 \mathrm{kPa}$. Comparisons of the FPG with three different PTB pressure standards operated in different pressure ranges - a pressure balance, a mercury manometer and a static expansion system - were performed using the cross-float method and by a direct comparison of the generated pressures. For the theoretical and experimental effective area, as well as for pressures generated by the FPG and the reference standards, all the results demonstrated full agreement within the expanded uncertainties of the standards.
\end{abstract}

\section{Section: RESEARCH PAPER}

Keywords: FPG; cross floating; effective area; pressure standard; experimental verification

Citation: Ahmed S. Hashad, Wladimir Sabuga, Sven Ehlers, Thomas Bock, Validation of a PTB force-balanced piston gauge primary pressure standard, Acta IMEKO, vol. 10, no. 1, article 1, March 2021, identifier: IMEKO-ACTA-10 (2021)-1-1

Section Editor: Momoko Kojima, NMIJ, Japan

Received April 22, 2020; In final form July 23, 2020; Published March 2021

Copyright: This is an open-access article distributed under the terms of the Creative Commons Attribution 3.0 License, which permits unrestricted use, distribution, and reproduction in any medium, provided the original author and source are credited.

Funding: This work was supported by the EMPIR-participating countries within EURAMET and the European Union and by the Egyptian study mission.

Corresponding author: Ahmed S. Hashad, e-mail: ahmed hashad84@hotmail.com

\section{INTRODUCTION}

Force-balanced piston gauges (FPGs) present an important class of piston gauge that enables pressure measurement from a few pascals to several tens of kilopascals. In these instruments, the force of the pressures acting on the piston of a pistoncylinder assembly (PCA) is measured by a high-accuracy balance. This force and the effective area $(A)$ of the PCA can be used to calculate the difference in the pressures above and below the piston [1], [2]. At PTB, a force-balanced piston gauge manufactured by Fluke Calibration (USA), model FPG8601 [3], [4], was characterised as a primary pressure standard by determining $A$ of the PCA from the PCA's dimensional properties [5], [6]. For this, a rarefied gas dynamics model was used to determine the pressure distribution of the gas flow inside the gap between the piston and the cylinder [7]-[9]. Three experiments were then performed to validate the PCA's effective area and the FPG pressures by comparing them with the pressures of three different standards. These standards have various operation principles and pressure ranges, as elaborated on below.

\section{EXPERIMENTS}

Experiments that can be used for the metrological characterisation of an FPG are described in, for example, [3], [10]-[17]. In the present study, experiments were specified that allowed the verification of the metrological characteristics obtained by the theoretical calculations. In the following sections, these experiments are described in detail. In all the experiments, a very low-pressure controller (VLPC), produced by Fluke Calibration, was used to stabilise the FPG measurement and lubrication pressures, unless otherwise stated. 


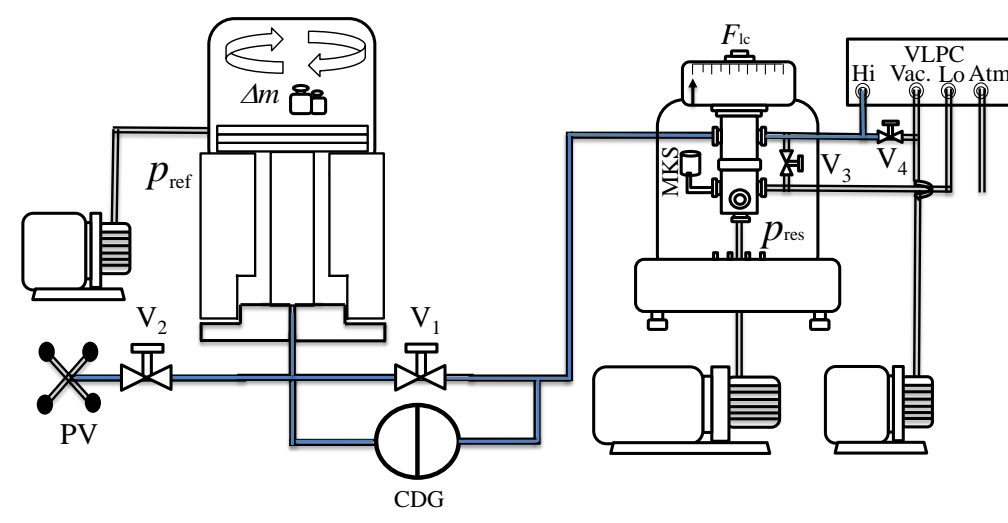

(a)

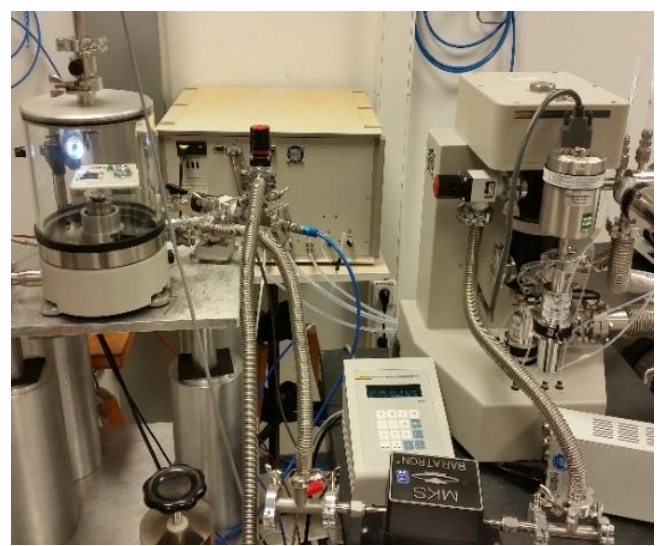

(b)

Figure 1. Experimental setup for cross floating between FPG and Ruska pressure balance: sketch of the whole setup (a) and life photo of the experiment (b).

\subsection{Effective area determination}

The FPG was calibrated against a Ruska pressure balance, model 2465A (gas-piston gauge), equipped with a PCA, serial no. TL1568, to determine the effective area of the FPG by the crossfloat method, as described in [17], [19]. The Ruska PCA has an effective area equal to $3.35666 \times\left(1 \pm 5.8 \cdot 10^{-6}\right) \mathrm{cm}^{2}$ (all uncertainties in this paper are given for $k=1$, unless otherwise specified). The effective area of this Ruska PCA is traceable to the state-of-the-art pressure balance that was used in the experiments on the redetermination of the Boltzmann constant [20]-[22] and is the German national pressure standard in the $180 \mathrm{kPa}$ to $7.5 \mathrm{MPa}$ range of absolute pressure. Both instruments, Ruska and FPG, were connected via the setup shown in Figure 1.

A class $E_{2}$ stainless-steel mass set was used to characterise the FPG balance [22]. A 10 torr capacitance diaphragm gauge (CDG) was placed in the measurement line to indicate the pressure difference between the two instruments. The pressures of both instruments were controlled to maintain the pressure difference measured with the CDG as close to zero as possible. The measurements were performed in gauge and absolute mode in the pressure ranges of $3 \mathrm{kPa}$ to $15 \mathrm{kPa}$ and $2 \mathrm{kPa}$ to $15 \mathrm{kPa}$, respectively. The higher starting pressure point of $3 \mathrm{kPa}$ in gauge mode was chosen due to the Ruska pressure balance's poor performance at lower pressures.

During the measurement, valve $V_{1}$, which separated both instruments, was closed, and valve $\mathrm{V}_{2}$ was opened. The pressure of the FPG was generated by the VLPC at the target pressure, and the variable pressure volume was used to generate the target pressure within the Ruska pressure balance. The VLPC then finely adjusted the pressure within the FPG until the CDG indication was close to zero. Finally, valve $V_{1}$ was opened and valve $V_{2}$ closed to allow a direct cross float between the FPG and the Ruska pressure balance.

From the experimental results, the effective area was calculated by means of equation (1),

$$
A=\frac{F_{\mathrm{lc}}}{\left(p_{\text {ref }}-p_{\text {res }}\right)[1+(\alpha+\beta)(t-20)]},
$$

in which $p_{\text {ref }}$ is the pressure of the reference standard, $p_{\text {res }}$ is the residual pressure on the lower side of the FPG PCA, which is equal to zero in gauge mode, $\alpha+\beta$ is the thermal expansion coefficient of the FPG PCA and $F_{\mathrm{lc}}$ is the force measured by the balance and corrected for buoyancy and lubrication pressurechange effects, as set out in [24] and shown in equation (2),

$$
F_{\mathrm{lc}}=\frac{m_{\mathrm{cal}}\left(1-\frac{\rho_{\mathrm{lub}}}{\rho_{\mathrm{m}}}\right) g}{N_{\mathrm{cal}}}\left(N+\delta N_{1}+\delta N_{2}+\delta N_{3}\right),
$$

where $m_{\text {cal }}$ is the true mass of the internal reference mass used for the balance's internal calibration, $N_{\text {cal }}$ is the reading of the balance when $m_{\text {cal }}$ is loaded, $N$ is the indication of the balance resulting from the pressure difference, $\delta N_{1}$ is the indication correction due to the lubricant gas-pressure variation, $\delta N_{2}$ is the indication correction due to the drag-force change and $\delta N_{3}$ is the indication correction due to the atmospheric-pressure variation.

The Ruska reference pressure ( $\left.p_{\text {R.ref }}\right)$ was calculated by means of equation (3),

$$
\begin{aligned}
p_{\text {R.ref }}= & \frac{g\left[m_{i}\left(1-\frac{\rho_{\mathrm{amb}}}{\rho_{i}}\right)+V\left(\rho_{\mathrm{l}}-\rho_{\mathrm{amb}}\right)\right]}{A_{\mathrm{r} 0}\left[1+\left(\alpha_{\mathrm{r}}+\beta_{\mathrm{r}}\right)(t-20)\right]} \\
& +p_{\text {R.rest }}+g h\left(\rho_{\mathrm{l}}-\rho_{\mathrm{amb}}\right),
\end{aligned}
$$

where $g$ is the local gravity acceleration, $m_{i}$ and $\rho_{i}$ are the masses and their densities, respectively, $\rho_{\mathrm{amb}}$ is the density of the ambient gas, $V$ is the piston's additional volume (which is required as a correction due to the buoyancy produced by the pressure-transmitting medium) with density $\rho_{\mathrm{l}}, A_{\mathrm{r} 0}$ is the effective area of the Ruska PCA, $\alpha_{\mathrm{r}}+\beta_{\mathrm{r}}$ is the thermal expansion coefficient of the Ruska PCA, $p_{\text {R.rest }}$ is the residual pressure in the Ruska bell jar and $h$ is the height difference between the reference levels of the pressure balance and the FPG.

\subsection{FPG vs mercury manometer}

In this experiment, the FPG was calibrated against the PTB mercury manometer (HgM), model 1025B made by Schwien Engineering (USA), which was modified at PTB and metrologically characterised, as described and validated in [25]-[26]. The design of this instrument is shown in Figure 2. This manometer comprises two vessels filled with mercury and connected by a flexible tube (A) with the ability to change the height of one of the vessels mechanically. The change in height of the movable vessel is measured by means of a laser interferometer (B). The height levels of the mercury's free surface in the vessels are controlled by capacitance measurements $\left(\mathrm{C}_{1}\right.$ and $\left.\mathrm{C}_{2}\right)$. In the experiments, the FPG was 


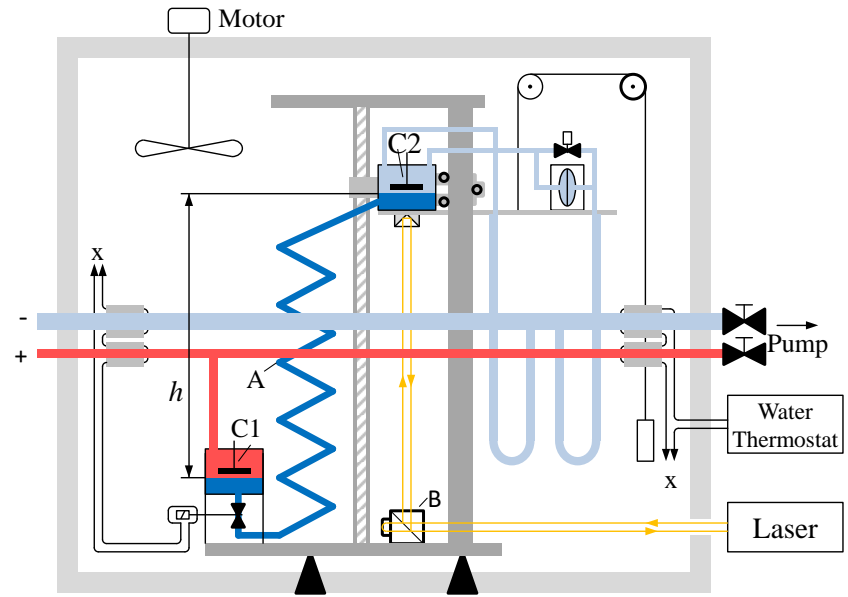

Figure 2. РTB mercury manometer $(\mathrm{HgM})$ with its reference $(-)$ and measurement $(+)$ pressure lines and climatisation temperature sensors $(x)$.

connected to the $\mathrm{HgM}$ directly; this is demonstrated in Figure 3 for measurements in gauge mode.

A CDG was placed between the two instruments to measure the pressure difference when changing the pressure in each instrument. All connections were thermally isolated to minimise the effect of ambient temperature changes even though all measurements were performed in an air-conditioned environment with a temperature stability within $0.3 \mathrm{~K}$. The measurements were carried out at a pressure range from $100 \mathrm{~Pa}$ to $15 \mathrm{kPa}$ in the gauge mode and from $1 \mathrm{kPa}$ to $15 \mathrm{kPa}$ in the absolute mode.

In the gauge-pressure mode, the VLPC was used as usual. At the beginning, zero pressures were set in both instruments, and both instruments were zeroed. After zeroing, pressures were generated separately. When generating the pressures, valve $V_{1}$, which separated both instruments, remained closed, and valve $V_{2}$ was open, allowing the $\mathrm{HgM}$ to be connected to its pressure generator. Thus, the target pressure was generated by the VLPC within the FPG and by the pressure generator within the $\mathrm{HgM}$. The height of the movable vessel was adjusted to make capacitances $C_{1}$ and $C_{2}$ equal (see Figure 2). Valve $V_{2}$ was then closed and, using the VLPC to finely adjust the pressure in the FPG, a close-to- zero indication of the CDG was reached. Next, bypath valve $V_{1}$ was opened to establish a direct connection and a pressure equilibrium between both systems. When equilibrium was achieved and all relevant indications became stable, their

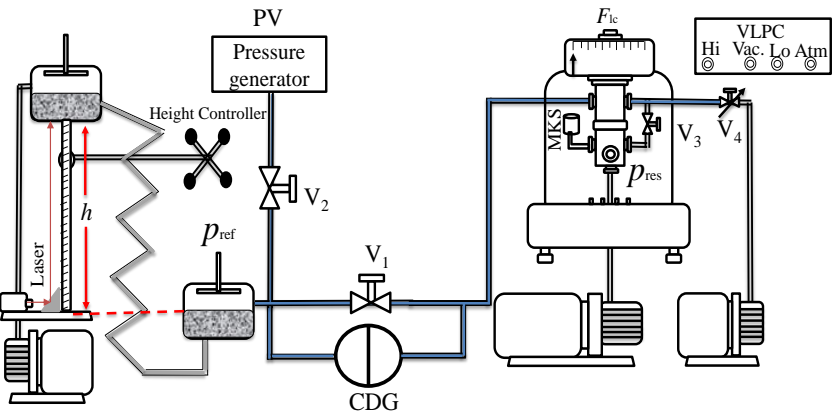

Figure 4. Experimental setup for cross floating between FPG and HgM in absolute mode.

values and all conditions were recorded.

In the absolute-pressure mode, the VLPC was replaced with valve $V_{4}$, which has a finely controlled opening and closing mechanism, behind which a turbopump was located (see Figure 4). The lubrication pressure was equal to $40 \mathrm{kPa}$, allowing the measurement pressure inside the FPG to be calculated by means of equation (4),

$$
p_{\mathrm{HgM}}=g h_{\mathrm{Hg}} \rho_{\mathrm{Hg}}+p_{\mathrm{M} . \mathrm{rest}}-g l \rho_{l},
$$

in which $h_{\mathrm{Hg}}$ is the height difference between the mercury levels in the two vessels, $l$ is the height difference between the reference level of the $\mathrm{HgM}$ and the mercury surface in the lower vessel, $\rho_{\mathrm{Hg}}$ is the density of mercury, $\rho_{l}$ is the density of the pressure-transmitting gas and $p_{\mathrm{M} . r e s t}$ is the residual pressure in the upper vessel of the HgM. The FPG pressure was calculated by means of equation (5) with two additional components, containing the height difference $h$ between the pressure reference levels of the FPG and $\mathrm{HgM}$ and the residual pressure $p_{\text {res. }}$ on the reference side of the FPG, which appears only in the absolute-pressure mode,

$$
p_{\mathrm{FPG}}=\frac{F_{\mathrm{lc}}}{A[1+(\alpha+\beta)(t-20)]}+p_{\text {res. }}-g h \rho_{l} .
$$

\subsection{FPG vs static expansion system}

The static expansion system (SES) [28], [29], see Figure 5, is a primary vacuum standard consisting of two stages of expansion, starting with two small volumes $\left(V L_{1}\right.$ and $\left.V L_{2}\right)$ and two intermediate volumes $\left(V L_{4}\right.$ and $\left.V L_{5}\right)$ and concluding with a

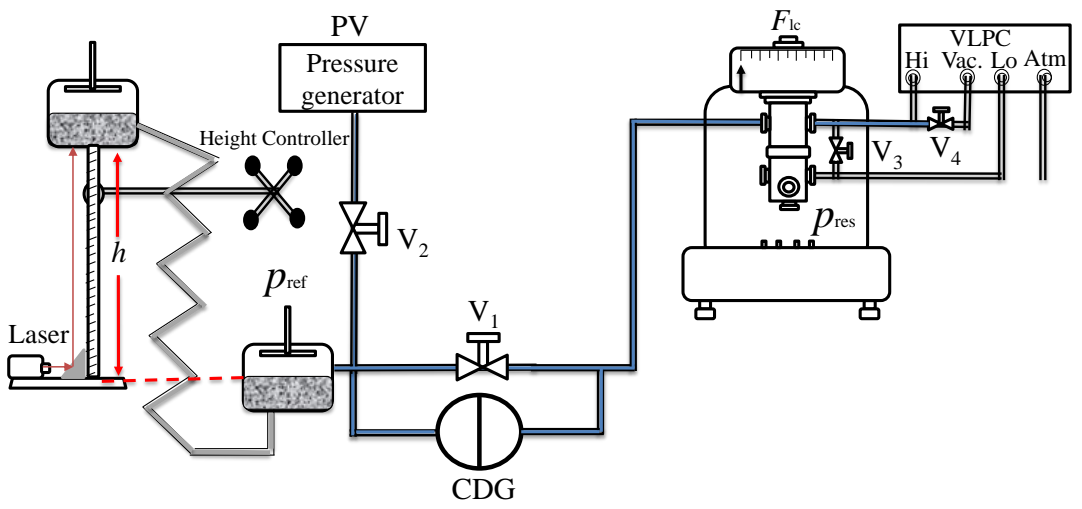

(a)

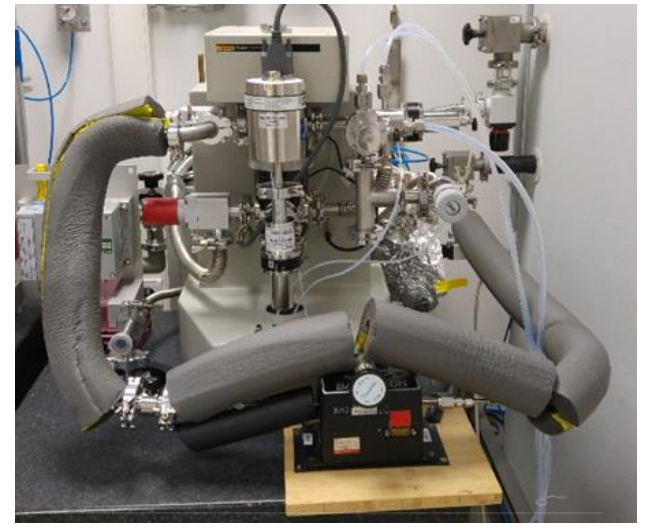

(b)

Figure 3. Experimental setup for cross floating between the FPG and the HgM in gauge-pressure mode: sketch of the whole setup (a) and life photo of the experiment (b) 


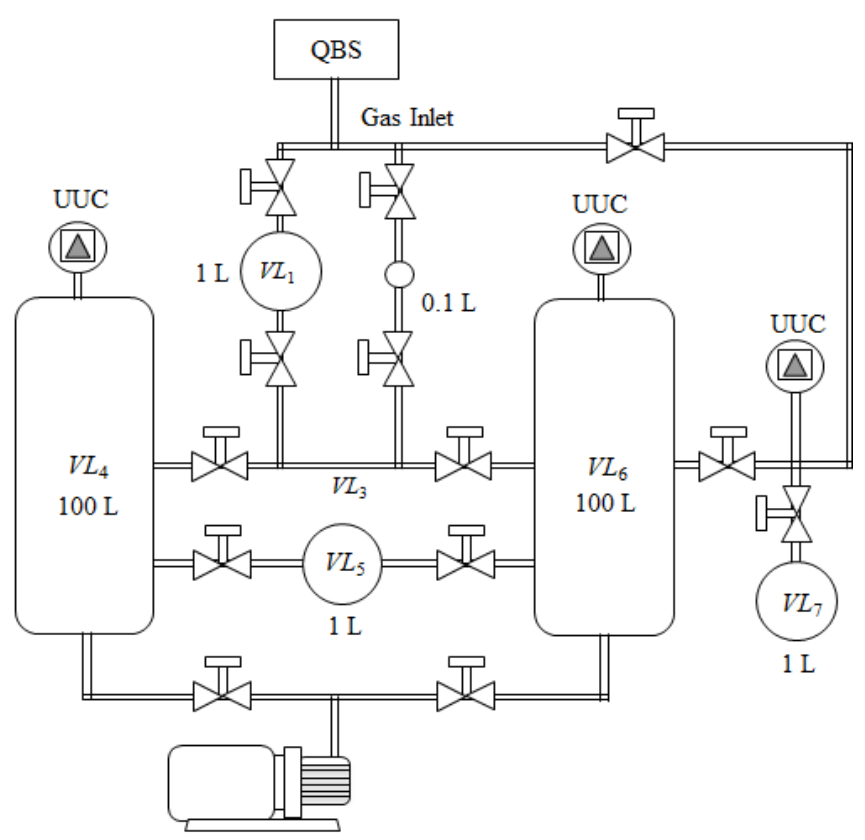

Figure 5. Design of SES.

calibration vessel $\left(V L_{6}\right)$ with a nominal volume of 100 litres. UUC is the unit under calibration. This set of volumes enables the realisation of the expansion ratios $f_{i}$, which are presented in Table 1.

A Quartz Bourdon Spiral (QBS) was used to control and measure the initial pressure value $p_{\text {fill }}$ when filling the first volume with the nitrogen gas in a range between $1 \mathrm{kPa}$ and $100 \mathrm{kPa}$. The final pressures produced by SES $p_{\text {S.ref }}$ in the range of $10 \mathrm{mPa}$ and $1 \mathrm{kPa}$ can be calculated by means of equation (6),

$$
p_{\text {S.ref }}=\frac{f_{i}^{\prime} p_{\text {fill }} T_{1}}{T_{0}\left(1+p_{\text {fill }} B /\left(R T_{0}\right)\right)}
$$

where $f_{i}^{\prime}$ is the corrected expansion ratio, taking the additional volume of the UUC into account, $T_{0}$ is the initial temperature before and $T_{1}$ the final temperature after the expansion, $B$ is the virial coefficient and $R$ is the gas constant.

The SES was used to verify the FPG in the low-pressure range from $3 \mathrm{~Pa}$ up to $300 \mathrm{~Pa}$. Because of the humidity of the nitrogen used inside the FPG and in order to keep the expansion volume $V L_{6}$ defined, a CDG, with a full range of $13.33 \mathrm{kPa}$ and $1 \mathrm{mPa}$ resolution, constantly separated the two systems, and its readings
Table 1. Expansion ratios of the SES shown in Figure 5.

\begin{tabular}{ccc}
\hline Symbol & Expansion ratio & $f$ \\
\hline$f_{1}$ & $\frac{V L_{1}}{V L_{1}+V L_{3}+V L_{6}}$ & $9.1732 \cdot 10^{-3}$ \\
$f_{2}$ & $\frac{V L_{2}}{V L_{2}+V L_{3}+V L_{6}}$ & $7.4231 \cdot 10^{-4}$ \\
$f_{3}$ & $\frac{V L_{1}}{V L_{1}+V L_{3}+V L_{4}+V L_{5}}$ & $9.1978 \cdot 10^{-3}$ \\
$f_{4}$ & $\frac{V L_{2}}{V L_{2}+V L_{3}+V L_{4}+V L_{5}}$ & $7.4378 \cdot 10^{-4}$ \\
$f_{5}$ & $\frac{V L_{5}}{V L_{5}+V L_{6}}$ & $9.170 \cdot 10^{-3}$ \\
\hline
\end{tabular}

were taken into consideration in the comparison results.

Figure 6 shows the experimental setup for the comparison of the FPG with the SES in absolute-pressure mode, where the SES is sketched simply with only two volumes. The VLPC was used to keep the pressure difference measured by the CDG as close to zero as possible. Initially, the QBS was used to set the pressure in $V L S_{1}$. Valves $\mathrm{V}_{2}$ and $\mathrm{V}_{6}$ were then opened to expand the gas into the larger volume $V L S_{2}$ and the tubes connected to the left side of the CDG before $\mathrm{V}_{1}$. The total volume was measured precisely. The SES pressure value was calculated according to equation (6).

\section{RESULTS}

The results of the effective area determination based on the FPG calibration against the Ruska pressure balance, which was described in Section 2.1, are presented in Table 2 and Figure 7 for both gauge and absolute-pressure operation modes.

Table 2. Experimental effective area $A$ and its standard uncertainty $u(A)$ $(k=1)$ of FPG at nominal pressures $p$ in gauge and absolute modes.

\begin{tabular}{lcccc}
\hline \multirow{2}{*}{$p / \mathrm{kPa}$} & \multicolumn{2}{c}{ Gauge mode } & \multicolumn{2}{c}{ Absolute mode } \\
\cline { 2 - 5 } & $A / \mathrm{cm}^{2}$ & $u(A) / \mathrm{cm}^{2}$ & $A / \mathrm{cm}^{2}$ & $u(A) / \mathrm{cm}^{2}$ \\
\hline 2 & & & 9.80592 & $4.8 \cdot 10^{-4}$ \\
3 & 9.80624 & $2.6 \cdot 10^{-4}$ & 9.80601 & $2.5 \cdot 10^{-4}$ \\
5 & 9.80620 & $1.6 \cdot 10^{-4}$ & 9.80600 & $1.8 \cdot 10^{-4}$ \\
6 & 9.80611 & $8.8 \cdot 10^{-5}$ & 9.80602 & $1.8 \cdot 10^{-4}$ \\
8 & 9.80607 & $1.1 \cdot 10^{-4}$ & 9.80601 & $1.5 \cdot 10^{-4}$ \\
10 & 9.80614 & $8.3 \cdot 10^{-5}$ & 9.80601 & $9.4 \cdot 10^{-5}$ \\
11 & 9.80617 & $7.5 \cdot 10^{-5}$ & 9.80606 & $1.0 \cdot 10^{-4}$ \\
13 & 9.80613 & $7.2 \cdot 10^{-5}$ & 9.80598 & $9.4 \cdot 10^{-5}$ \\
15 & 9.80610 & $8.0 \cdot 10^{-5}$ & 9.80599 & $8.7 \cdot 10^{-5}$ \\
\hline
\end{tabular}

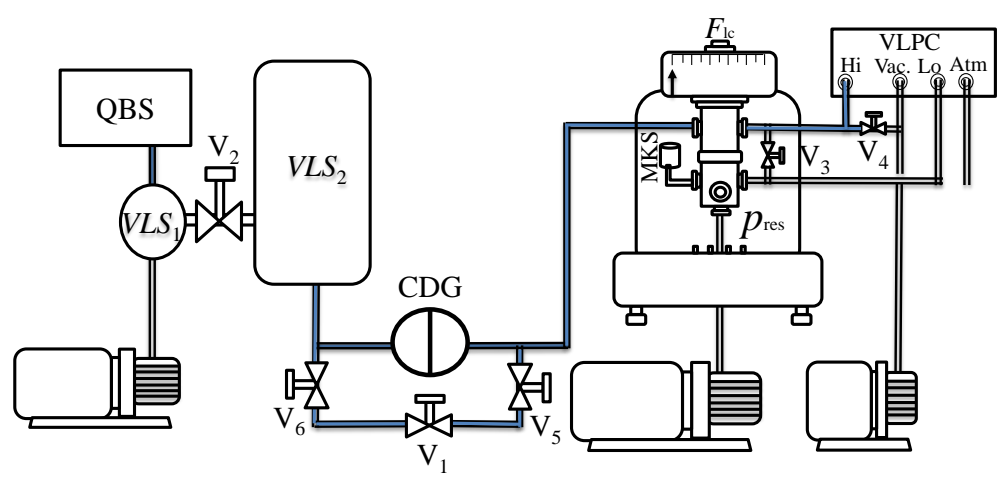

(a)

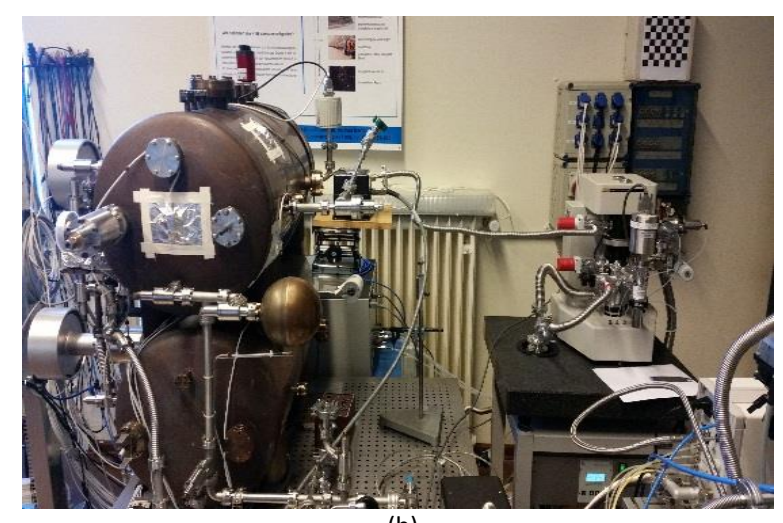

(b)

Figure 6. Experimental setup for comparison between FPG and SES: sketch of the whole setup (a) and life photo of the experiment (b). 


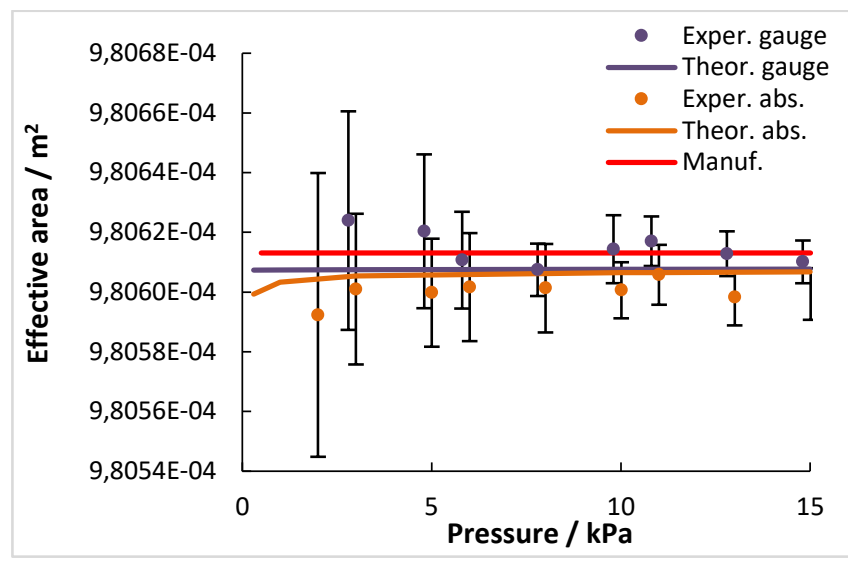

Figure 7. Experimental (Exper.), RGD-calculated (Theor.) and manufacturer's (Manuf.) effective areas of the FPG in absolute (abs.) and gauge (gauge) modes.

In Figure 7, these experimental results are shown together with the results of a theoretical determination of FPG's effective area based on the dimensional properties of the PCA, calculated using rarefied gas dynamics (RGD) methods and with a relative standard uncertainty of $4 \cdot 10^{-6}$. Figure 7 also shows the effective area reported by the manufacturer with a relative standard uncertainty of $13 \cdot 10^{-6}$. All experimental and theoretical effective areas agree well in that the experimental standard uncertainties are lower than typical FPG uncertainties [29].

The results of the pressure comparison between the FPG and the $\mathrm{HgM}$ are presented for gauge mode and absolute mode in Figure 8 and Figure 9, respectively.

The pressure differences measured in gauge mode have a more pronounced scatter than in absolute mode since the gaugepressure measurements were affected by the instability of the atmospheric pressure. Because the time constants of the FPG and the $\mathrm{HgM}$ differed considerably, the temporal reactions of the two instruments to the ambient pressure variations were significantly different, leading to the pressure differences observed in the experiment. In addition, the large volume of the tubes connecting the FPG to the HgM made it difficult to control the pressure during the measurements. Despite this, almost all pressure differences are covered by the expanded uncertainty $(k=2)$ of the $\mathrm{HgM}$.

On the contrary, in absolute-pressure mode, the measurements were free of the ambient pressure effect, and the results were characterised by a much lower scatter of the pressure differences (see Figure 9). The differences in the pressures

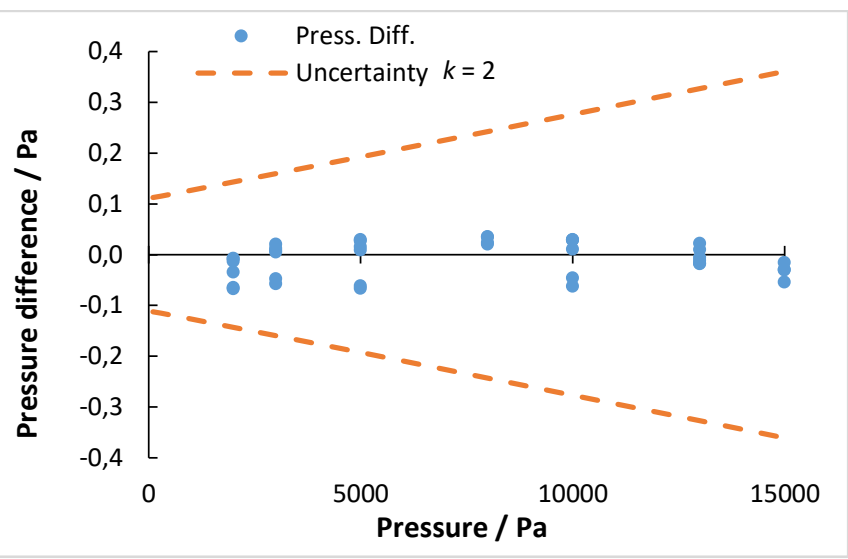

Figure 9. Differences in absolute pressures measured with FPG and HgM.

measured with the FPG and those measured with the HgM are much smaller than the expanded uncertainty of the HgM [26], which demonstrates the consistency of the two pressure standards.

In the lowest pressure range of the FPG operation (from $3 \mathrm{~Pa}$ to $300 \mathrm{~Pa}$ ), consistency was verified via a comparison with the SES in the absolute-pressure mode. The results of the comparison of the two pressure standards in terms of the pressure differences and their uncertainties are shown in Figure 10 .

Above $30 \mathrm{~Pa}$, the pressure differences agree with the standard uncertainty $(k=1)$ of the SES. At lower pressures, the scatter of the pressure differences is greater than the standard uncertainty of the SES, but it is in agreement with the expanded uncertainty $(k=2)$ and allows conclusions to be drawn about the consistency of the two standards at $10 \mathrm{mPa}$.

\section{CONCLUSIONS}

For the theoretical and experimental effective area of the PCA, as well as for pressures generated by the FPG and the three reference standards based on different operation principles (the pressure balance, the mercury manometer and the static expansion system), all the results demonstrate full agreement within the expanded uncertainties $(k=2)$ of the three standards. Thus, the FPG, which has already been characterised as a primary pressure standard, is validated experimentally. For the pressure range from $3 \mathrm{~Pa}$ to $15 \mathrm{kPa}$, the FPG standard uncertainty is presently estimated as $10 \mathrm{mPa}+6 \cdot 10^{-6} \times p$.

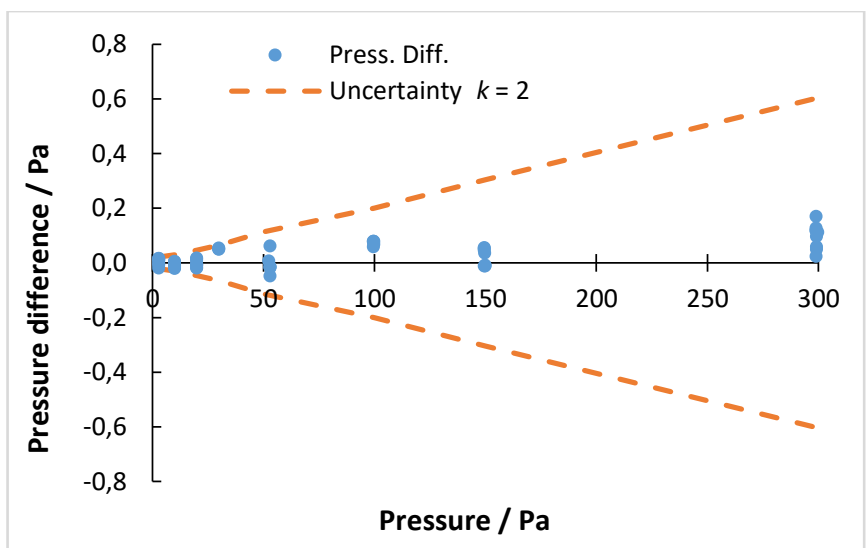

Figure 10. Differences in absolute pressures measured with FPG and SES. 


\section{ACKNOWLEDGEMENT}

The first author wishes to thank the Egyptian study mission for their support and the funding of his scientific mission at PTB.

The research presented in this article was carried out within EMPIR, which is jointly funded by the EMPIR-participating countries within EURAMET and the European Union.

\section{REFERENCES}

[1] A. Ooiwa, Development of a highly stable air piston pressure gauge with non-rotational piston, Flucom'85, Tokyo, Japan, 2 September 1985, pp. 959-964.

[2] A. Ooiwa, Novel nonrotational piston gauge with weight balance mechanism for the measurement of small differential pressures, Metrologia 30 (1993/94), pp. 607-610. DOI: $10.1088 / 0026-1394 / 30 / 6 / 012$

[3] P. Delajoud, M. Girard, A force balanced piston gauge for very low gauge and absolute pressure, Proc. of the $10^{\text {th }}$ Intern. Metrology Congress, St Louis, France, 22 - 25 October 2001.

[4] R. Haines, M. Bair, Application of a new method for the automated calibration of very low gauge and absolute pressures in a commercial calibration laboratory, Meas. Sci. Conf., Anaheim CA, USA, January 2002. Online [Accessed 25 March 2021] https://pdfs.semanticscholar.org/f2a5/253226166ab949ceb5b1d cad39fd41b861a4.pdf

[5] A. Hashad, S. Ehlers, O. Jusko, W. Sabuga, Characterization of a force-balanced piston gauge as a primary pressure standard, Measurement 131 (2019), pp. 723-729.

DOI: 10.1016/i.measurement.2018.09.01

[6] S. Naris, N. Vasileiadis, D. Valougeorgis, A. Hashad, W. Sabuga, Computation of the effective area and associated uncertainties of non-rotating piston gauges FPG and FRS, Metrologia 56 (2019), pp. $1-10$

DOI: $10.1088 / 1681-7575 /$ aaee 18

[7] F. M. Sharipov, V. D. Seleznev, Rarefied gas flow through a long tube at any pressure ratio, J. Vac. Sci. \& Technol. A 12 (1994), pp. 2933-2935.

DOI: $10.1116 / 1.578969$

[8] V. A. Titarev, E. M. Shakhov, Rarefied gas flow through a long circular pipe into vacuum, AIP Conf. Proc. 1501 (2012), pp. $465-472$.

DOI: $10.1063 / 1.4769572$

[9] F. Sharipov, Rarefied Gas Dynamics. Fundamentals for Research and Practice, Wiley-VCH, Berlin, 2016, ISBN 978-3-527-41326-3.

[10] P. Otal, J. C. Legras, Metrological characterization of a new standard, in absolute and gauge pressure modes, in the range $1 \mathrm{~Pa}$ to $15000 \mathrm{~Pa}$, Metrologia 42 (2005), pp. 216-219.

DOI: $10.1088 / 0026-1394 / 42 / 6 /$ S15

[11] M. Rantanen, S. Semenoja, Results on the effective area of the FPG-type digital piston manometer of MIKES, Metrologia 42 (2005), pp. 165-168.

DOI: $10.1088 / 0026-1394 / 42 / 6 / S 03$

[12] M. Rantanen, S. Semenoja, G. Peterson, J. Busk, Low gauge pressure comparisons between MIKES, Metrosert and FORCE Technology, Report on EUROMET Project 921, MIKES Publication J2/2007. Online [Accessed 25 March 2021] https://www.vttresearch.com/sites/default/files/pdf/MIKES/2 007-J2.pdf

[13] D. A. Vijayakumar, O. Prakash, R. K. Sharma, Establishment of a force balanced piston gauge for very low gauge and absolute pressure measurements at NPL, India, J. Phys. Conf. Ser. 390 (2012) 012013

DOI: $10.1088 / 1742-6596 / 390 / 1 / 012013$

[14] R. Haines, M. Bair, A method of traceability for a FPG8601 force balanced piston gauge to define pressures in the range from $1 \mathrm{~Pa}$ to $15 \mathrm{kPa}$ in gauge and absolute measurement modes, Proc. of the XIX IMEKO World Congress, Lisbon, Portugal, 6-11 September 2009, pp. 2071-2076. Online [Accessed 25 March 2021]
https://www.imeko.org/publications/wc-2009/IMEKO-WC2009-TC16-298.pdf

[15] J. H. Hendricks, D. A. Olson, 1-15,000 Pa Absolute mode comparisons between the NIST ultrasonic interferometer manometers and non-rotating force-balanced piston gauges, Measurement 43 (2010), pp. 664-674.

DOI: $10.1016 /$ i.measurement.2009.12.031

[16] J. H. Hendricks, D. A. Olson, NIST experience with non-rotating force-balanced piston gauges for low pressure metrology, Proc. of the XIX IMEKO World Congress, Lisbon, Portugal, 6-11 September 2009. Online [Accessed 25 March 2021] https://www.imeko.org/publications/wc-2009/IMEKO-WC2009-TC16-549.pdf

[17] Z. Krajičček, M. Bergoglio, K. Jousten, P. Otal, W. Sabuga, S. Saxholm, D. Pražák, M. Vičar, Final report on EURAMET.M.P-K4.2010: Key and supplementary comparison of national pressure standards in the range $1 \mathrm{~Pa}$ to $15 \mathrm{kPa}$ of absolute and gauge pressure, Metrologia 51 (2014) Tech. Suppl. 07002.

DOI: $10.1088 / 0026-1394 / 51 / 1 \mathrm{~A} / 07002$

[18] J. Tesar, P. Repa, D. Prazak, Z. Krajicek, L. Peksa, The new method of traceability of a force-balanced piston gauge used as primary vacuum standard, Vacuum 76 (2004), pp. 491-499. DOI: $10.1016 /$ i.vacuum.2004.04.008

[19] J.-S. Chang, S.-G. Lee, Uncertainty evaluation of low pressure by means of cross-float, Proc. of the XX IMEKO World Congress, Busan, Rep. of Korea, 9-14 September 2012. Online [Accessed 25 March 2021] https://www.imeko.org/publications/wc-2012/IMEKO-WC2012-TC16-P7.pdf

[20] W. Sabuga, T. Priruenrom, R. Haines, M. Bair, Design and evaluation of pressure balances with $1 \cdot 10^{-6}$ uncertainty for the Boltzmann constant project, PTB-Mitteilungen 121 (2011), pp. 256-259.

[21] W. Sabuga, Pressure measurements in gas media up to 7.5 MPa for the Boltzmann constant redetermination, PTB-Mitteilungen 121 (2011), pp. 247-255.

[22] T. Zandt, W. Sabuga, C. Gaiser, B. Fellmuth, Measurement of pressures up to $7 \mathrm{MPa}$ applying pressure balances for dielectricconstant gas thermometry, Metrologia 52 (2015), pp. S305-S313. DOI: $\underline{10.1088 / 0026-1394 / 52 / 5 / \text { S305 }}$

[23] Non-automatic weighing instruments, Part 1: Metrological and technical requirements - Tests, International Recommendation OIML R 76-1, Edition 2006 (E). Online [Accessed 25 March 2021] https://www.oiml.org/en/files/pdf r/r076-1-e06.pdf

[24] FPG8601 ${ }^{\mathrm{TM}} /$ VLPC $^{\mathrm{TM}}$, Operation and maintenance manual, DH Instruments, a Fluke company, 2007.

[25] H. Bauer, Die Darstellung der Druckskala im Bereich von 0.01 bar bis 2 bar durch das Quecksilber-Normalmanometer der PTB, PTB-Mitteilungen 89 (1979), pp. 248-255. Online [Accessed 25 March 2021]

[26] J. Jäger, Use of a precision mercury manometer with capacitance sensing of the menisci, Metrologia 30 (1993/94), pp. 553-558. DOI: $10.1088 / 0026-1394 / 30 / 6 / 002$

[27] M. Perkin, A. Picard, M. Lecollinet, K. Fen, M. Sardi, A. Miiller, A. Agarwal, M. Jescheck, C. Wüthrich, Final report on CCM key comparison CCM.P-K2: pressure $(10 \mathrm{kPa}$ to $120 \mathrm{kPa})$ absolute mode, Metrologia 45 (2008) Tech. Suppl. 07002. DOI

[28] T. Bock, H. Ahrendt, K. Jousten, Reduction of the uncertainty of the PTB vacuum pressure scale by a new large area non-rotating piston gauge, Metrologia 46 (2009), pp. 389-396. DOI: $10.1088 / 0026-1394 / 46 / 5 / 001$

[29] W. Steckelmacher, The calibration of vacuum gauges, Vacuum 37 (1987), pp. 651-657.

DOI: $10.1016 / 0042-207 X(87) 90051-0$

[30] M. Bair, P. Delajoud, Typical pressure measurement uncertainty defined by an FPG8601 force balanced piston gauge, DH Instruments, a Fluke company, Technical Note 2090TN05, Rev. 26 March 2004. 